\title{
Spin-labeling Investigations of Polyamides and Polyesters
}

\author{
PERTTI TÖRM ÄL Ä, KATI SILVENNOINEN and \\ J. J OHAN LIN D B ER G
}

Department of Wood and Polymer Chemistry, University of Helsinki, Helsinki, Finland

\begin{abstract}
Polyamides from sebacoyl chloride and hexamethylenediamine and polyesters from sebacoyl chloride and dihydroxybenzenes with 3-carboxy-2,2,5,5-tetramethylpyrroline-1-oxyl end groups were prepared. The ESR spectra of solid polymers indicated that the mobility of end group radicals is severely restricted. The ESR spectra of polyamides and polyesters were measured in $m$-cresol and chloroform solutions, respectively. The hard sphere radii of dissolved polymers were calculated by means of the Stokes-Einstein expression. A loosely arranged random-coil orientation of polymer chains in these solvents was indicated.
\end{abstract}

Spin-labeling technique has been used widely to study structural problems

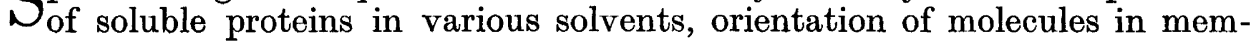
branes and structures of molecular aggregates in solvents., ${ }^{1,2}$ It is well known that the electron spin resonance (ESR) spectrum of free nitroxyl radicals in a polymer matrix consists of a triplet and is very similar to the spectrum of the same radicals in a high viscous liquid. ${ }^{3}$ On the other hand the ESR spectra of nitroxyl polymers consist of a single exchange-narrowed line. ${ }^{4}$ It can be concluded that spin-labeling of polymer end groups reflects the intermolecular relations in the polymer matrix..$^{13}$

We wish to report here the preparation and ESR spectra of condensation polymers of sebacoyl chloride with hexamethylenediamine, hydroquinone, catechol, and resorcinol with 3-carboxy-2,2,5,5-tetramethylpyrroline-1-oxyl end groups. The ESR spectra were analysed according to Edelstein et al. ${ }^{5}$

\section{EXPERIMENTAL}

Materials. 3-Carboxy-2,2,5,5-tetramethylpyrroline-1-oxyl (I) was prepared according to Rozantsev and Krinitskaya. ${ }^{6}$

3-Chloroformyl-2,2,5,5-tetramethylpyrroline-1-oxyl (II) was prepared according to Krinitskaya et al.? from (I) just before each polymerization to get a reactive compound for end group labeling. 
<smiles>CC1(C)C(=O)N([O])C1(C)C</smiles>

(I)<smiles>CC1(C)C=C(C(=O)Cl)C1(C)O</smiles>

(II)

Spin-labeled polyamides from sebacoyl chloride and hexamethylenediamine (IIIa-IIId). Polyamide IIIa was prepared by modifying the interfacial polycondensation method of Beaman et al. ${ }^{8}$ To a solution of $0.007 \mathrm{~mol}$ of sebacoyl chloride in $50 \mathrm{ml}$ of symtetrachloroethane $0.0002 \mathrm{~mol}$ of II in $2 \mathrm{ml}$ of benzene was added. The mixture was cooled to $0^{\circ} \mathrm{C}$. To the vigorously stirred solution 0.021 mol hexamethylenediamine in $25 \mathrm{ml}$ of water was added. The mixture was stirred $30 \mathrm{~min}$ at $0^{\circ} \mathrm{C}$. The product was washed several times with water and ether and extracted with acetone until the ESR activity of the sample remained constant. The polymer was dried in vacuum overnight. The polyamides IIIb-IIId were prepared in the same manner by using various amounts of radical II (to IIIb $0.0004 \mathrm{~mol}$ of II was added, to IIIc 0.0018 and to IIId, $0 \mathrm{~mol}$, respectively). Because radical II has only one covalently active group it causes the stopping of the condensation reaction. Thus also various amounts of radical in the reaction mixture give various molecular weights in the products. The yields were $60-70 \%$. The structural formula of the paramagnetic end of IIIaIIIc is given below.

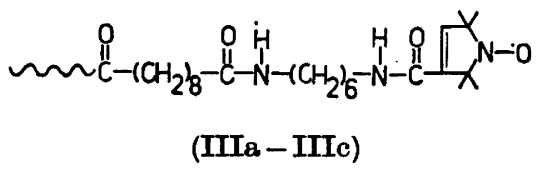

Spin-labeled polyesters from sebacoyl chloride and dihydroxybenzenes (IVa-IVc). Polyester TVa was prepared by adding to $28.5 \mathrm{ml}$ of benzene-pyridine mixture $(15: 1 \mathrm{v} / \mathrm{v})$ $0.005 \mathrm{~mol}$ of sebacoyl chloride, $0.0005 \mathrm{~mol}$ of radical II in $1.5 \mathrm{ml}$ of benzene and $0.005 \mathrm{~mol}$ of hydroquinone. The reaction vessel was sealed and the solution was shaken at $24^{\circ} \mathrm{C}$ for $3 \mathrm{~h}$ and was left overnight. The precipitates were removed by centrifugation and the solution was poured into a large amount of chilled methanol-water mixture $(19: 1 \mathrm{v} / \mathrm{v})$. The precipitated polymer was centrifugated, dried and dissolved in a small amount of benzene-pyridine mixture $(15: 1 \mathrm{v} / \mathrm{v})$. The solution was precipitated with chilled methanol-water mixture. This dissolving-precipitating procedure was repeated so many times that the ESR activity of the sample remained constant. The product was dried in vacuum overnight. The other polyesters were prepared in the same way with the exception that instead of hydroquinone in IVb catechol and in IVc resorcinol were used. The yields were $10-15 \%$. The structural formula of paramagnetic end of IVa-IVc is given below.

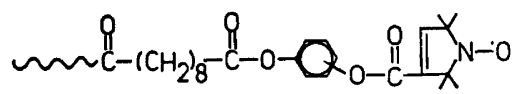

$(\mathrm{IVa}-\mathrm{IVc})$

Molecular weights. The polymeric nature of each polyamide prepared (IIIa-IIId) was characterized in terms of its intrinsic viscosity in $m$-cresol at $30^{\circ} \mathrm{C}$. The molecular weights of polyesters prepared (IVa-IVc) were measured using a vapour pressure osmometer (Perkin-Elmer Modell 115) in chloroform at $40^{\circ} \mathrm{C}$.

Acta Chem. Scand. 25 (1971) No. 7 
ESR measurements. The ESR spectra were obtained from solid and dissolved polymers on a Varian E-4 spectrometer operating at a microwave frequency of $9.5 \mathrm{GHz}$. The magnetic field was modulated with an amplitude less than one-fourth of the linewidth. The measurements were made at $297^{\circ} \mathrm{K}$ and at $336^{\circ} \mathrm{K}$ using a thermostat. Its accuracy was $\pm 1^{\circ}$.

The isotropic ESR spectra of polyamides and polyesters were obtained at $336^{\circ} \mathrm{K}$ in diethylene glycol and in chloroform solutions, respectively. The concentration of nitroxyl radicals in solution was $\sim 10^{-5} \mathrm{M}$.

To get the parameters of the spin Hamiltonian, the glass spectra of the samples were measured at $100^{\circ} \mathrm{K}$.

\section{RESULTS AND DISCUSSION}

The determined molecular weights are given in Table 1. The molecular weights of polyamides become smaller when the amount of nitroxide radicals in the beginning becomes greater. The relation between radical concentration and the $[\eta]$ of polyamides was linear in this concentration range.

Table 1.

\begin{tabular}{ccccc}
\hline Compound & $\begin{array}{c}\text { Nitroxyl radicals } \\
\text { added (mol) }\end{array}$ & {$[\eta]$} & $\begin{array}{c}\text { Molecular } \\
\text { weight }\end{array}$ \\
\hline Polyamide IIId & 0.0000 & 0.85 & 9100 \\
" & IIIa & 0.0002 & 0.50 & 5150 \\
" IIIb & 0.0004 & 0.45 & 4700 \\
& IIIc & 0.0018 & 0.38 & 4000 \\
Polyester & IVa & 0.0005 & & 2600 \\
" IVb & IVc & 0.0005 & & 4200 \\
" & 0.0005 & & 4200 \\
\hline
\end{tabular}

ESR Spectra of solid polymers. The glass spectrum at $100^{\circ} \mathrm{K}$ of polyamide IIIa is given in Fig. 1. The other glass spectra looked principally alike. An analysis based on the assumed axial symmetry of the spectra was carried out

Fig. 1. The ESR spectrum of polyamide IIIa at $100^{\circ} \mathrm{K}$.

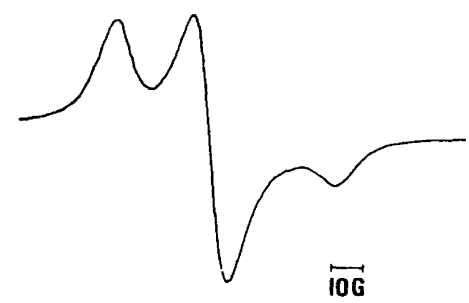

according to Edelstein et al..$^{5}$ It is known that the two outermost peaks of the glass spectrum of nitroxyl radicals are caused by the parallel transitions of the nuclear spin states $\mathrm{M}_{\mathrm{I}}= \pm 1$. This gives the possibility to determine $g_{11}$, and $A_{11}$. Thus the experimental isotropic quantities $\langle g\rangle$, and $\langle A\rangle$, obtained by the above mentioned method, together with the known relationships Acta Chem. Scand. 25 (1971) No. 7 
$\langle g\rangle=\frac{1}{3}\left(g_{11}+2 g_{1}\right)$ and $\langle\mathrm{A}\rangle=\frac{1}{3}\left(A_{11}+2 A_{1}\right)$ give the values for the parameters of the spin, which are collected in Table 2.

Table 2.

\begin{tabular}{lcccc}
\hline Compound & $A_{\| 1}(\mathrm{G})$ & $A_{\perp}(\mathrm{G})$ & $g_{\|}$ & $g_{\perp}$ \\
\hline${\text { Polyamides IIIa }-\mathrm{IIIc}^{a}}^{a}$ & 34.8 & 5.2 & 2.0050 & 2.0080 \\
Polyesters IVa-IVc $^{b}$ & 33.0 & 5.0 & 2.0048 & 2.0071 \\
\hline
\end{tabular}

${ }^{a}$ Isotropic $\langle A\rangle=15.1 \mathrm{G},\langle g\rangle=2.0070$

${ }^{b}$ Isotropic values $\langle A\rangle=14.9 \mathrm{G},\langle g\rangle=2.0067$

The ESR spectra of polyamides and polyesters at $297^{\circ} \mathrm{K}$ and $336^{\circ} \mathrm{K}$ are given in Figs. 2 and 3. They are highly anisotropic, so the correlation times of nitroxyl radicals are in the region of slow rotations. The spectra of low molecular weight polymers (Fig. 2, IIIc and Fig. 3, IVa) indicate further strong spin-spin intermolecular interaction. In Table 3 are given the values of peakto-peak separation of the outermost lines of the spectra.

It can be seen from the peak-to-peak separations that the mobility of labeled polyamide molecules at constant temperature becomes greater when the molecular weight of the sample becomes smaller. These spectra, compared with earlier results for polymers with more weak intermolecular interactions, ${ }^{9}$

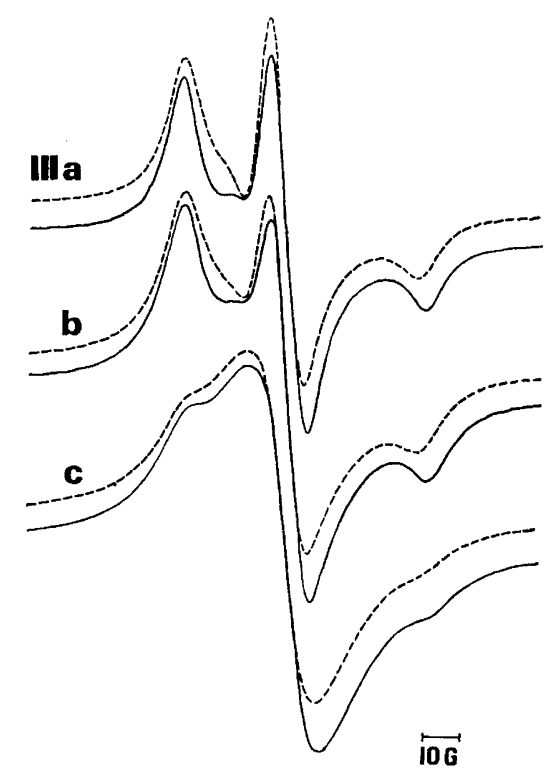

Fig. 2. The ESR spectra of spin-labeled polyamides ( $\left.297^{\circ} \mathrm{K},---336^{\circ} \mathrm{K}\right)$.

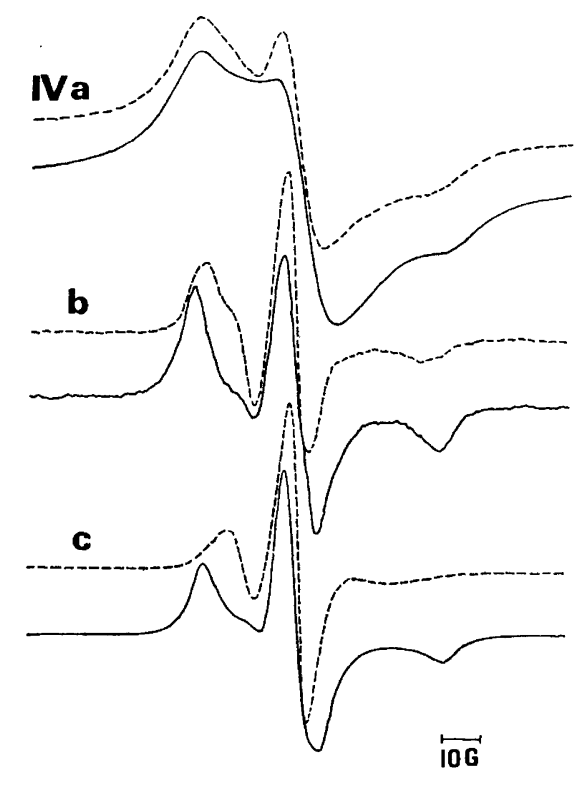

Fig. 3. The ESR spectra of spin-labeled polyesters ( $297^{\circ} \mathrm{K},--336^{\circ} \mathrm{K}$ ).

Acta Chem. Scand. 25 (1971) No. 7 
Table 3.

\begin{tabular}{cccccc}
\hline \multirow{2}{*}{ Compound } & Molecular & \multicolumn{3}{c}{ Peak-to-peak separation (G) } \\
weight & & $100^{\circ} \mathrm{K}$ & $297^{\circ} \mathrm{K}$ & $336^{\circ} \mathrm{K}$ \\
\hline \multirow{2}{*}{ Polyamide IIIa } & 5150 & 69.6 & 66.6 & 64.4 \\
" & IIIb & 4700 & 69.6 & 66.2 & 63.0 \\
" & IIIc & 4000 & 69.6 & 66.0 & 60.0 \\
Polyester & IVa & 2600 & 66.0 & 62.0 & 42.0 \\
" & IVb & 4200 & 66.0 & 63.0 & 52.0 \\
" & IVc & 4200 & 66.0 & 61.0 & 59.0 \\
\hline
\end{tabular}

seem to indicate that strong intermolecular bonds restrict markedly the motions of covalently bonded radicals. Further it is evident that the velocity of the increase of motional freedom as a function of temperature is dependent on the steric conditions of polymers ( $c f$. polymers IVa-IVc of Table 3).

Spin-labeled polymers in solution. The spin-labeled polyamides and polyesters were dissolved in $m$-cresol and chloroform, respectively, to study the effective Stokes radii of molecules. The solutions were diluted so much that the dissolved polymers did not more affect the microviscosity of solutions. The Stokes-Einstein expression for $\tau_{\mathrm{c}}$,

$$
\tau_{\mathrm{c}}=\frac{4 \pi \eta a^{3}}{3 k T}
$$

predicts that at constant temperature, $\tau_{\mathrm{c}}$ is a linear function of $\eta$. By measuring $\tau_{c}$ and $\eta$ it is possible to estimate the equivalent hard-sphere radius of the tumbling molecule. In Fig. 4 are given the ESR spectra of polyamide IIIa
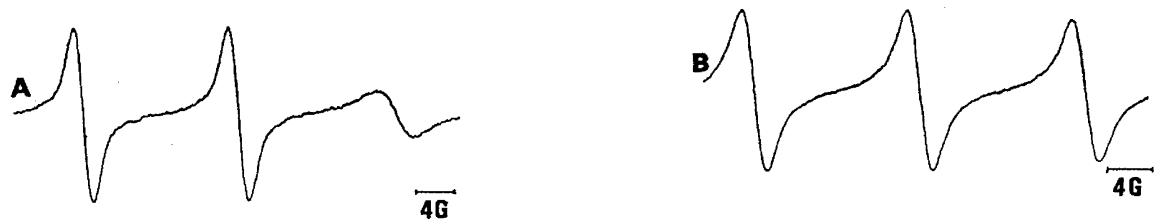

Fig. 4. A. Polyamide IIIa in $m$-cresol. B. Polyester IVa in chloroform $\left(T=297^{\circ} \mathrm{K}\right)$.

and polyester IVa in $m$-cresol and chloroform, respectively, at $297^{\circ} \mathrm{K}$. The other solution ESR-spectra looked principally alike. The correlation times were calculated according to Barratt et al. ${ }^{10}$ by means of the expression

$$
\tau_{\mathrm{c}}=\frac{\delta v(\mathrm{M}=-1)+\delta v(\mathrm{M}=+1)}{\delta v(\mathrm{M}=0)}\left\{\frac{15 \pi \sqrt{3} \delta v(\mathrm{M}=0)}{8 b \Delta \gamma \bar{H}}\right\}
$$

where $\delta v(\mathrm{M}=0, \pm 1)$ are the measured spectral line widths,

$$
\Delta \gamma=\beta / \hbar\left(g_{11}-g_{\perp}\right) \text { and } b=\frac{4 \pi}{3}\left(A_{11}-A_{\perp}\right) \text {. In Table } 4 \text { are given the values of }
$$
$\tau_{\mathrm{c}}$ and the corresponding hard sphere radii $(a)$ of polymer molecules calculated Acta Chem. Scand. 25 (1971) No. 7 
Table 4.

\begin{tabular}{cccc}
\hline \multicolumn{2}{c}{ Compound } & $\tau_{\mathrm{c}}(\mathrm{s})$ & $a(\AA)$ \\
\hline Polyamide & IIIa & $5.3 \times 10^{-9}$ & 6.9 \\
& IIIb & $5.1 \times 10^{-9}$ & 6.6 \\
IIIc & $5.0 \times 10^{-9}$ & 6.5 \\
Polyester & IVa & $1.2 \times 10^{-10}$ & 6.0 \\
& IVc & $1.4 \times 10^{-10}$ & 7.0 \\
& IVc & $1.3 \times 10^{-10}$ & 6.5 \\
\hline
\end{tabular}

by the expression (1). The effective Stokes radii of Table 4 are considerably greater than the corresponding ones, measured by the same method, for free radicals (I) in $m$-cresol $(a=1.9 \AA)$ and chloroform $(a=2.9 \AA)$. These values are smaller than the real radii of the molecules as was stated earlier. ${ }^{5}$ The effective Stokes radii of polymers are much smaller than expected from the molecular weights of polymers. This indicates that the end group radicals still possess a great amount of motional freedom relative to the polymer backbone and that a loosely arranged random coil orientation of polymer chains is present.

According to the theory of Kivelson ${ }^{11}$ the line width of hyperfine multiplets of an ESR spectrum is influenced by the hyperfine anisotropy, the $g$-factor anisotropy and the correlation time. Assuming a Lorenzian line shape, the expression is

$$
\Delta H_{\mathrm{mI}}=\left(a_{1}+a_{2} \mathrm{~m}_{\mathrm{I}}+a_{3} \mathrm{~m}_{\mathrm{I}}^{2}\right)
$$

where $\Delta H_{\mathrm{mI}}$ is the peak-to-peak line width of the absorption derivative, and

$$
\begin{gathered}
a_{1}=\tau_{\mathrm{c}}\left[\frac{4}{45}\left(\beta \hbar^{-1} \Delta g B_{0}\right)^{2}+\frac{3 b^{2}}{20}\right]+\frac{\tau_{\mathrm{c}}}{1+\omega_{0}{ }^{2} \tau_{\mathrm{c}}^{2}}\left[\frac{1}{15}\left(\beta \hbar^{-1} \Delta g B_{0}\right)^{2}+\frac{7 b^{2}}{20}\right]+K \\
a_{2}=-\tau_{\mathrm{c}}\left[b \beta \hbar^{-1} \Delta g B_{0}\right]\left[\frac{4}{45}+\frac{1}{5\left(1+\omega_{0}{ }^{2} \tau_{\mathrm{c}}{ }^{2}\right)}\right] \\
a_{3}=\tau_{\mathrm{c}} \frac{b^{2}}{8}-\left[\frac{\tau_{\mathrm{c}}}{1+\omega_{0}^{2} \tau_{\mathrm{c}}^{2}}\right]\left[\frac{b^{2}}{40}\right]
\end{gathered}
$$

Here $\Delta g=\left(g_{11}-g_{\perp}\right), B_{0}$ is the applied field in Gauss, $K$ represents the contribution of relaxation processes to the linewidth which are independent of $\tau_{\mathrm{c}}$ and $b=2 / 3 \times\left(A_{11}-A_{1}\right)$. By insertion of $\tau_{\mathrm{c}}$ in eqns. 4-6 and by using the experimental value [(derivative height $) \times(\text { derivative width })^{2}=$ constant $]$ for $\Delta H_{\mathrm{mr}}$ it is possible to calculate $K$. Thus for polyamides a value of $K=0.30 \times 10^{7} \mathrm{~s}^{-1}$ is obtained. For polyesters it is $\sim 0.60 \times 10^{7} \mathrm{~s}^{-1}$ at $297^{\circ} \mathrm{K}$. These data are of the same order of magnitude as earlier results for free nitroxyl radicals in solutions. ${ }^{12}$ 


\section{REFERENCES}

1. Ingham, J. D. Rev. Macromol. Chem. 3 (1968) 279.

2. Griffith, O. H. and Waggoner, A. S. Acc. Chem. Res. 2 (1969) 17.

3. Rozantsev, E. G. Free Nitroxyl Radicals, Plenum Press, New York 1970, p. 172.

4. Griffith, O. H., Keana, J. F. W., Rottschaefer, S. and Warlick, T. A. J. Am. Chem. Soc. 89 (1967) 5072.

5. Edelstein, N., Kwok, A. and Maki, A. H. J. Chem. Phys. 41 (1969) 179.

6. Rozantsev, E. G. and Krinitskays, C. A. Tetrahedron 21 (1965) 491.

7. Krinitskaya, L. A., Buchachenko, A. C. and Rozantsev, E. G. Zh. Organ. Khim. 2 (1966) 1301.

8. Beaman, R. G., Morgan, D. W., Koller, C. R., Wittbecker, L. and Magat, E. E. J. Polymer Sci. 40 (1959) 289.

9. Törmälä, P., Martinmaa, J., Silvennoinen, K. and Vaahtera, K. Acta Chem. Scand. 24 (1970) 3066.

10. Barratt, M. D., Green, D. K. and Chapman, D. Biochim. Biophys. Acta 152 (1968) 20.

11. Kivelson, D. J. Chem. Phys. 33 (1960) 1107.

12. Lenk, R., Theobald, J. G. and Uebersfeld, J. Compt. Rend. 261 (1965) 4071.

13. Bullock, A. T., Butterworth, J. H. and Cameron, G. G. European Polymer J. 7 (1971) 445.

Received December 29, 1970. 